\title{
Si Doping of GaN in Hydride Vapor-Phase Epitaxy
}

\author{
E. RICHTER, ${ }^{1,3}$ T. STOICA, ${ }^{2}$ U. ZEIMER,${ }^{1}$ C. NETZEL, ${ }^{1}$ M. WEYERS,${ }^{1}$ \\ and G. TRÄNKLE ${ }^{1}$ \\ 1.-Ferdinand-Braun-Institut, Gustav-Kirchhoff-Str. 4, 12489 Berlin, Germany. 2.-Peter \\ Grünberg Institute (PGI-9), Forschungszentrum Jülich, and Jülich-Aachen Research Alliance \\ (JARA), 52425 Jülich, Germany. 3.—e-mail: eberhard.richter@fbh-berlin.de
}

Growth of GaN boules by hydride vapor-phase epitaxy (HVPE) is very attractive for fabrication of GaN substrates. Use of dichlorosilane as a source for Si doping of bulk GaN is investigated. It is shown that no tensile strain is incorporated into mm-thick, Si-doped GaN layers on sapphire substrates if the threading dislocation density is previously reduced to $2.5 \times 10^{7} \mathrm{~cm}^{-2}$ or below. High-quality GaN layers with electron densities up to $1.5 \times 10^{19} \mathrm{~cm}^{-3}$ have been achieved, and an upper limit of about $4 \times 10^{19} \mathrm{~cm}^{-3}$ for Si doping of GaN boules was deduced considering the evolution of dislocations with thickness. A 2-inch, Si-doped GaN crystal with length exceeding $6 \mathrm{~mm}$ and targeted Si doping of about $1 \times 10^{18} \mathrm{~cm}^{-3}$ is demonstrated.

Key words: HVPE, GaN, bulk, Si doping, defects, strain

\section{INTRODUCTION}

There is a great need for suitable substrates for fabrication of current group III nitride devices such as violet-blue laser diodes (LDs) and white lightemitting diodes (LEDs) as well as for realization of, e.g., green LDs and high-brightness LEDs with high performance or other future device concepts. ${ }^{1}$ Such substrates should allow for epitaxial growth of differently oriented device layer structures with low defect densities, i.e., threading dislocation density (TDD) below $10^{6} \mathrm{~cm}^{-2}$ and negligible stacking fault density (SFD). It has been shown that appropriate native GaN substrates with high quality can be obtained by cutting and subsequent polishing of slices from thick GaN crystals grown by hydride vapor-phase epitaxy (HVPE). ${ }^{2,3}$ For optoelectronic applications, a sufficiently high $n$-type conductivity and high transparency of the substrates are also desired. The most commonly used donor dopant in epitaxy of III-V compounds is silicon. However, it has been observed that use of $\mathrm{Si}$ for doping is accompanied by incorporation of tensile strain and hence reduced critical layer thickness for crack formation during epitaxial growth of GaN layers. ${ }^{4-6}$ Early explanations suggested point $\operatorname{defects}^{4,7}$ or

(Received August 16, 2012; accepted November 26, 2012; published online December 20, 2012) coalescence of crystallites at the growth front ${ }^{6}$ as the reason for the observed tensile strain, because the size difference between dopant and host atoms was found to be small. A breakthrough was achieved in terms of a quantitative model that explained the reduction of compressive strain in Si-doped $\mathrm{Al}_{x} \mathrm{Ga}_{1-x} \mathrm{~N}$ layers by inclination of edge dislocations, referred to as an "effective climb" mechanism. However, the underlying mechanism at the growing surface, i.e., either silicon nitride micromasking of dislocation $\operatorname{cores}^{9}$ or Fermi-level-induced formation of vacancy jogs attached to dislocation cores, ${ }^{10}$ is still under controversial debate. Even recently, focusing on GaN layers with high TDD, contradicting statements have been published which propose Ge as a beneficial $n$-type dopant to circumvent tensile strain $^{11}$ or not. ${ }^{12}$ However, avoiding incorporation of tensile strain and the resulting crack formation is essential for boule-like growth of $\mathrm{GaN}$ in HVPE. Use of elemental silicon, ${ }^{13}$ dichlorosilane (DCS) ${ }^{14-16}$ and more recently $\mathrm{GeCl}_{4}{ }^{16}$ has been reported for successful $n$-type doping of GaN layers grown by HVPE. The aim of this study is to determine whether $\mathrm{Si}$ is a suitable dopant for boule-like HVPE growth of GaN of several mm length at high growth rates exceeding $300 \mu \mathrm{m} / \mathrm{h}$ starting on GaN/ sapphire templates. ${ }^{17}$ Taking into account that the amount of tensile strain increases with the $\mathrm{Si}$ concentration as well as with the TDD, ${ }^{8-10}$ the idea 
pursued here is to grow first a GaN layer without $\mathrm{Si}$ incorporation to reduce the TDD, followed by growth of the Si-doped GaN boule.

\section{EXPERIMENTAL PROCEDURES}

The starting GaN/sapphire templates consisted of non-intentionally doped (n.i.d.) GaN layers of about $3.5 \mu \mathrm{m}$ thickness grown by metalorganic vaporphase epitaxy (MOVPE) on 2-inch, $c$-plane sapphire substrates of $430 \mu \mathrm{m}$ thickness in an Aixtron 2600 G3 multiwafer reactor. The TDD of these templates is estimated to be about $1 \times 10^{9} \mathrm{~cm}^{-2}$ from x-ray diffraction measurements. ${ }^{17}$ The HVPE growth was performed in an Aixtron vertical HVPE reactor ${ }^{18}$ using metallic $\mathrm{Ga}$, gaseous hydrogen chloride, ammonia, a nitrogen/hydrogen gas mixture as carrier gas, and 3\% DCS solved in hydrogen as dopant source, which was added to the carrier gas near the group III gas inlet. The HVPE growth was conducted at $200 \mathrm{hPa}$. N.i.d. GaN layers were grown at a rate of about $450 \mu \mathrm{m} / \mathrm{h}$ at $990^{\circ} \mathrm{C}$, whereas it was found more favorable to grow the Si-doped layers at a rate of about $400 \mu \mathrm{m} / \mathrm{h}$ at $1010^{\circ} \mathrm{C}$. A series of five samples was fabricated with a n.i.d. GaN layer grown for $30 \mathrm{~min}(225 \mathrm{~nm})$ first, followed by rampup temperature and doping gas for $5 \mathrm{~min}$, and subsequently growth of a Si-doped GaN layer for $150 \mathrm{~min}(1 \mathrm{~mm})$ with addition of $0.5 \mathrm{sccm}, 1 \mathrm{sccm}$, $2 \mathrm{sccm}, 4 \mathrm{sccm}$, or $8 \mathrm{sccm}$ of the dopant gas. These layers separated from the sapphire substrates spontaneously after cooldown due to the large difference of the thermal expansion coefficients. Samples of $9 \mathrm{~mm} \times 9 \mathrm{~mm}$ were diced, and adjacent specimens were selected for secondary-ion mass spectrometry measurements of silicon and oxygen. The backside of the samples was polished to sample thicknesses below $1 \mathrm{~mm}$ in order to remove the n.i.d. GaN layer completely. Hall-effect measurements were performed at room temperature using small welded In contacts and a Keithley K 925 B measurement setup with $B=0.36 \mathrm{~T}$. X-ray diffraction investigations were performed using a Philips X'Pert Pro in double-axis geometry for symmetric and skew-symmetric rocking curves and in triple-axis geometry in order to determine lattice constants from $\Theta / 2 \Theta$-scans at 004, 006, 105, 106, 204, and 206 reflections. ${ }^{19} \mu$-Raman measurements were performed in $z(x x)-z$ backscattering geometry using excitation wavelength of $532 \mathrm{~nm}$ and a Dilor XY500 triple monochromator with a charge-coupled device (CCD). Cathodoluminescence (CL) measurements were performed to determine the TDD by counting the dark spots. Two additional growth experiments were conducted. In the first, the growth time of the n.i.d. GaN layer was reduced from 30 min to only $75 \mathrm{~s}$ (less than $10 \mu \mathrm{m}$ ) to check the impact of the n.i.d. GaN layer. In the second, the doping was adjusted to a free carrier concentration of about $1 \times 10^{18} \mathrm{~cm}^{-3}(0.75 \mathrm{sccm}$ of $3 \%$ DCS $)$ and the growth time of the Si-doped layer was prolonged to $15 \mathrm{~h}$ to obtain a GaN boule.

\section{RESULTS AND DISCUSSION}

The oxygen concentration in the five samples of the doping series was found to be below the detection limit of SIMS, i.e., below $2 \times 10^{16} \mathrm{~cm}^{-3}$. In Fig. 1 the results of SIMS determination of the $\mathrm{Si}$ concentration and the electron concentration from Hall-effect measurements are compared. It can be seen that the electrical activation of the incorporated $\mathrm{Si}$ is as high as $90 \%$ over the whole doping range with electron densities from $8.7 \times 10^{18} \mathrm{~cm}^{-3}$ to $1.5 \times 10^{19} \mathrm{~cm}^{-3}$. The Si uptake increases linearly with the dopant flow.

In Fig. 2, the Hall mobilities are depicted for the different electron concentrations. These mobilities in doped layers are higher than those we reported in Ref. 15 for n.i.d. GaN layers on sapphire with oxygen acting as major donor. Thin layers usually exhibit a much higher TDD of above $10^{9} \mathrm{~cm}^{-2}$, but Si-doped GaN layers with similar thickness on sapphire also showed higher mobilities. ${ }^{15}$ According to Ref. 20, the mobilities of O-doped GaN layers are remarkably affected by $\mathrm{V}_{\mathrm{Ga}}-\mathrm{O}_{\mathrm{N}}$-related edge dislocation scattering when the edge dislocation density exceeds $10^{8} \mathrm{~cm}^{-2}$. The different nature of the dopants $\mathrm{Si}, \mathrm{Ge}$, and $\mathrm{O}$ is discussed in Refs. 21,22. It is argued that donors incorporated on Ga sites could reduce the Coulomb scattering of $\mathrm{V}_{\mathrm{Ga}}$ acceptors and thus enhance the carrier mobility. It can be seen that the mobilities obtained for our Si-doped layers are similar to those published by Oshima et al. ${ }^{16}$ for thick, $n$-type doped GaN layers grown on GaN substrates with TDD on the order of $10^{6} \mathrm{~cm}^{-2}$ and thus represent the state of the art.

No cracks were observed in the samples of the $\mathrm{Si}$ doping series. To evaluate the impact of the $\mathrm{Si}$ doping on the crystal structure, the GaN lattice constants were determined by high-resolution x-ray diffraction. The results are shown in Fig. 3 together with data which represent the ideal lattice constants of state-of-the-art GaN. ${ }^{23}$ The scales of the

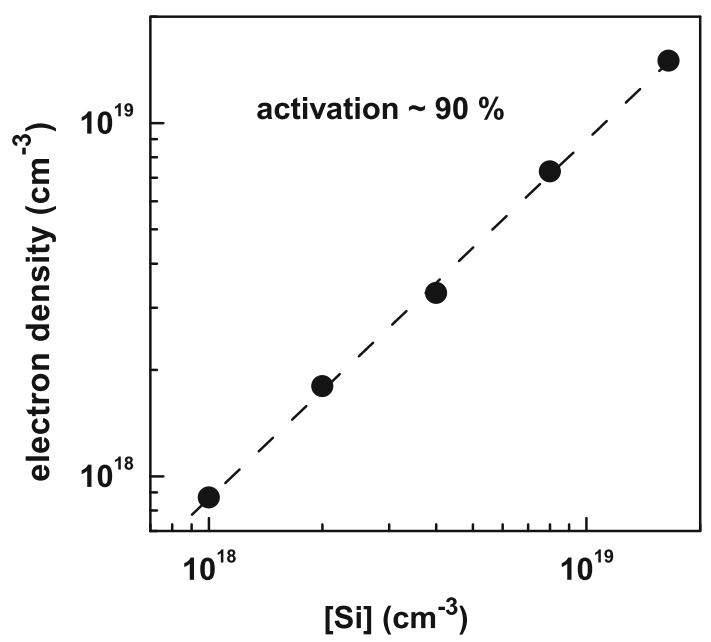

Fig. 1. Comparison of the free electron density measured by Hall effect and the incorporated Si concentration measured by SIMS. 


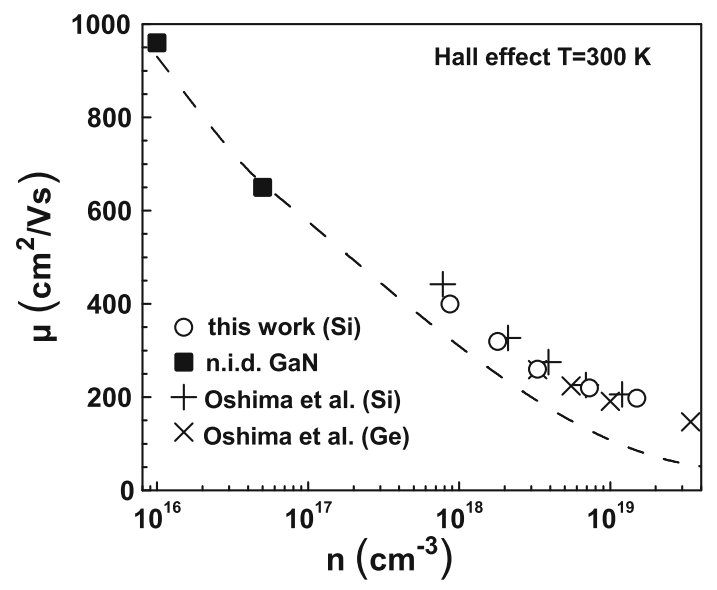

Fig. 2. Mobility versus carrier concentration from Hall effect measured for the series of Si-doped samples (open symbols). The dashed line depicts an interpolation between numerous values of thin n.i.d. GaN layers on sapphire. ${ }^{15}$ Two data points (squares) obtained from freestanding n.i.d. GaN samples grown in the vertical reactor used in this study are inserted. For comparison, data published by Oshima et al. for Si- and Si/Ge-doped GaN layers grown by HVPE on $\mathrm{GaN}$ substrates are shown. ${ }^{16}$

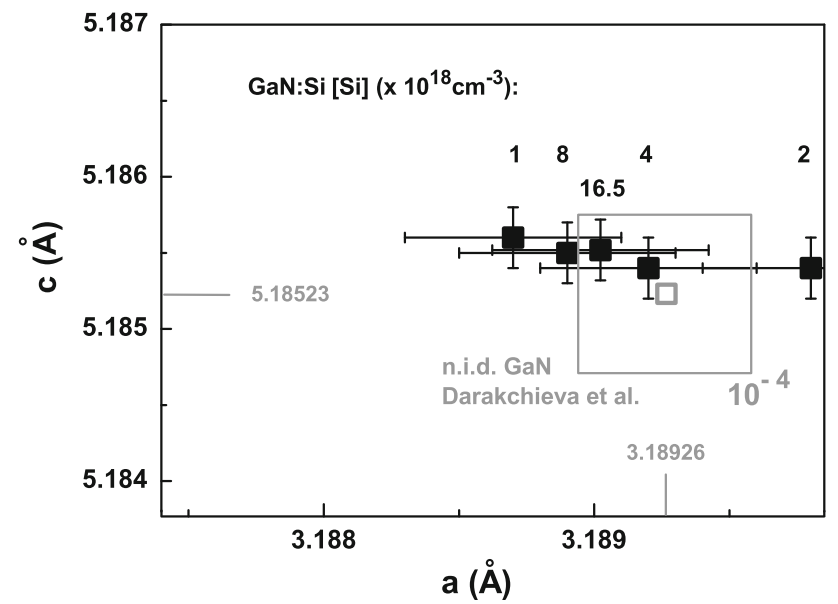

Fig. 3. Chart of the lattice constants $a$ and $c$ for state-of-the-art n.i.d. bulk GaN after Ref. 23 with an indicated target region for the strain of less than $10^{-4}$ and the results from high-resolution $\mathrm{x}$-ray diffraction measurements of the samples of the doping series with Si concentration measured by SIMS.

diagram are similar to the ones in Ref. 23 , because it was shown there that high-quality bulk GaN grown by different methods can exhibit substantial differences in the lattice parameters. However, for fabrication of epiready GaN substrates, it is important to meet the ideal lattice constants $a$ and $c$ within a narrow range. A window corresponding to a deviation of $10^{-4}$ in the lattice constants is indicated as a useful target region. It can be seen that the obtained data still show considerable scatter, especially in the in-plane lattice constant $a$, but taking into account the error bars they are all very close to the target. In addition, the individual data points in Fig. 3 do not show a systematic change, indicating

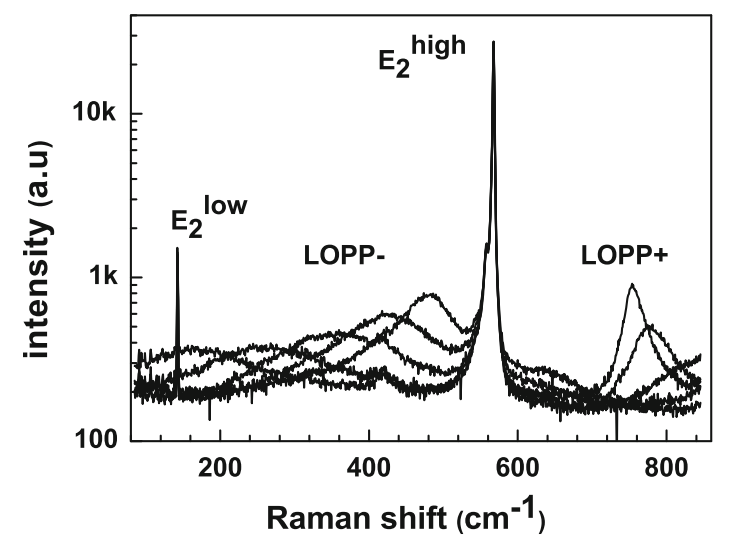

Fig. 4. Raman spectra recorded in backscattering geometry on the samples of the Si doping series.
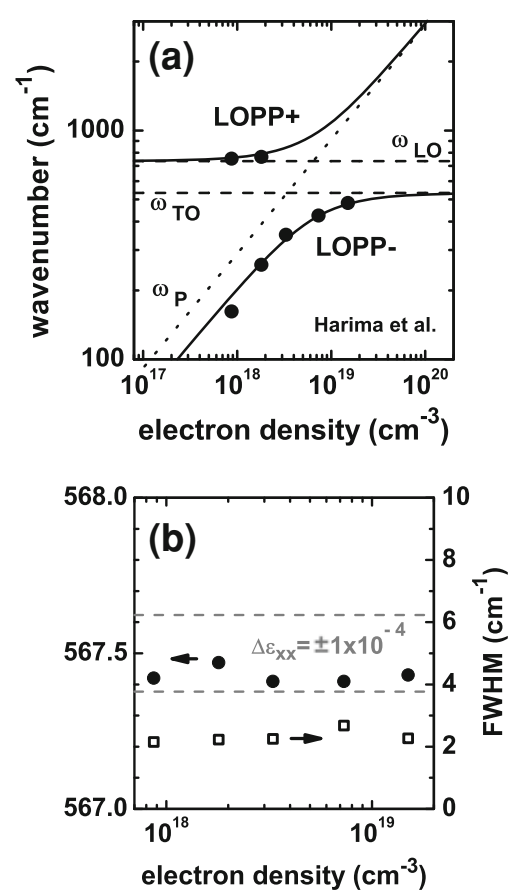

Fig. 5. Raman shifts of the longitudinal phonon-plasmon modes together with calculated positions after Harima et al. ${ }^{24}$ in (a) and Raman shifts and full-width at half-maximum (FWHM) of the $E_{2}^{\text {high }}$ modes in (b) as observed for the different electron densities of the Si-doped samples in Fig. 4.

that the scatter may be due to remaining uncertainties in our measurements.

To clarify the origin of the scatter in the measured lattice parameters, Raman spectra (Fig. 4) were recorded. The spectra show phonon-like resonances indicated as $E_{2}^{\text {low }}$ and $E_{2}^{\text {high }}$ and longitudinal optical phonon-plasmon (LOPP- and LOPP+) modes. These features of the spectra are analyzed for the different samples, and the corresponding Raman shifts are depicted versus the electron densities in more detail in Fig. 5a, b.

In Fig. 5a, good agreement between the calculated $^{24}$ and experimental data for the coupled phonon-plasmon modes is observed, which indicates a 
high potential of two-dimensional (2D) or threedimensional (3D) Raman imaging for further spatially resolved investigations of $n$-type GaN. In Fig. $5 \mathrm{~b}$, negligible fluctuation of the $E_{2}^{\text {high }}$ position is revealed with narrow full-width at half-maximum (FWHM) values. The latter is an indicator of high structural quality of the crystal, whereas the position of the $E_{2}^{\text {high }}$ sensitively depends on the in-plane lattice constant $a$. A Raman wavenumber shift of $\Delta v\left(E_{2}^{\text {high }}\right)$ of about $-0.123 \mathrm{~cm}^{-1}$ per in-plane strain $\Delta \varepsilon_{x x}$ of 0.0001 is given in Ref. 25 . The $E_{2}^{\text {high }}$ wavenumbers of the Si-doped GaN samples are all close to $567.5 \mathrm{~cm}^{-1}$, which is the phonon frequency of strain-free $\mathrm{GaN}^{3}{ }^{3}$ Hence, the differences of the in-plane lattice constants of the samples compared with state-of-the-art n.i.d. GaN are below $10^{-4}$. For the highest $\mathrm{Si}$ concentration, the Raman measurement (not shown here) was also performed on the sample backside, but no change of the $E_{2}^{\text {high }}$ phonon frequency could be observed; i.e., no residual strain gradient from the backside to the sample surface is indicated.

The FWHM of x-ray diffraction rocking curves (RC-FWHM) at the 002 and 302 reflection were

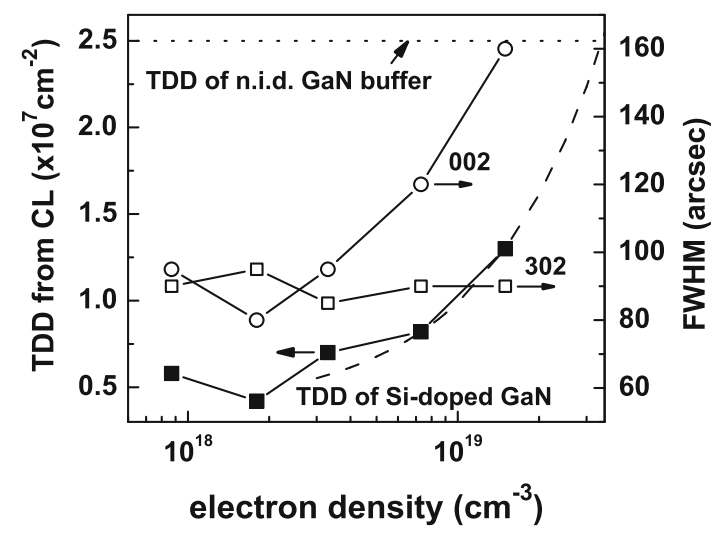

Fig. 6. TDD from CL images and RC-FWHM at 002 and 302 reflection versus electron density. The dotted line indicates the TDD level after growth of the n.i.d. GaN buffer layer. The dashed line denotes extrapolated TDD for 1-mm-thick GaN layers with higher Si doping, which crosses the dotted line at electron density of about $4 \times 10^{19} \mathrm{~cm}^{-3}$. determined to evaluate differences in screw, mixed, and edge dislocation densities between the samples. For comparison, dark spot densities from CL images were used to estimate the overall TDD at the surface of the samples. The TDD of the n.i.d. GaN layer was estimate from CL images from a sample backside to be $2.5 \times 10^{7} \mathrm{~cm}^{-2}$. The other data are depicted in Fig. 6.

It can be seen that, for all the samples, the TDD is reduced during the growth of the Si-doped GaN layer of $1 \mathrm{~mm}$ thickness; however, the reduction mechanism is less effective with increasing $\mathrm{Si}$ uptake above $[\mathrm{Si}]>2 \times 10^{18} \mathrm{~cm}^{-3}$, and from simple extrapolation no reduction of TDD is expected for doping levels above $4 \times 10^{19} \mathrm{~cm}^{-3}$. Interestingly, from the dependences of the RC-FWHM, it can be concluded that this less effective reduction is related to a lower reactivity of screw dislocations because the RC-FWHM at the 002 reflection shows a similar dependence whereas the RC-FWHM at the 302 reflection is unaffected by the Si uptake, indicating unperturbed reduction of edge and mixed dislocations. ${ }^{26}$ These findings suggest that $\mathrm{Si}$ is a useful doping source in the investigated doping range but limited to the mid $10^{19} \mathrm{~cm}^{-3}$ range, above which no reduction of the TDD with thickness is expected under our growth conditions.

Optical micrographs of the surfaces of the layers with the lowest and highest $\mathrm{Si}$ doping of $1 \times 10^{18} \mathrm{~cm}^{-3}$ and $1.65 \times 10^{19} \mathrm{~cm}^{-3}$ are depicted in Fig. 7a, b as examples of the samples of the $\mathrm{Si}$ doping series. The surfaces are very similar, with some tendency for refined morphological features with increasing doping level, although no indication of crack formation during growth was found. In an additional experiment, the thickness of the n.i.d. GaN layer was reduced to about $9 \mu \mathrm{m}$ before the growth of a 1-mm-thick GaN layer with the highest Si doping of $1.65 \times 10^{19} \mathrm{~cm}^{-3}$ in order to verify the role of the TDD reduction in the n.i.d. GaN layer. The TDD after the growth of $9 \mu \mathrm{m} \mathrm{GaN}$ is still near $1 \times 10^{19} \mathrm{~cm}^{-2}$. The corresponding surface shown in Fig. 7c on the microscopic scale is similar to the corresponding one in Fig. $7 \mathrm{~b}$ but with multiple cracks at and below the surface, mostly along $\{1-100\}$.
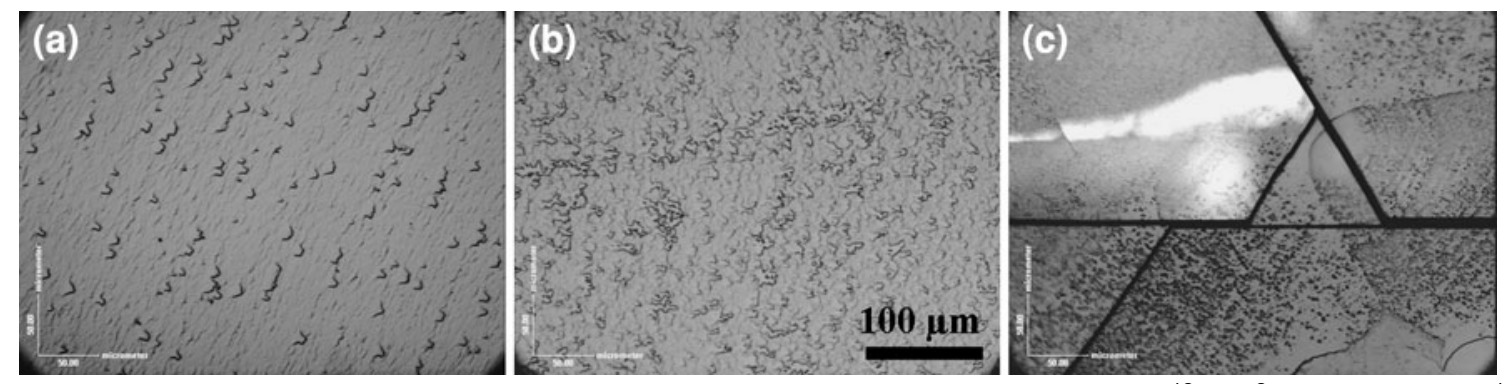

Fig. 7. Optical micrographs of surfaces of 1-mm-thick, Si-doped GaN layers with Si content [Si] of $1 \times 10^{18} \mathrm{~cm}^{-3}(\mathrm{a}) \mathrm{and} 1.65 \times 10^{19} \mathrm{~cm}^{-3}(\mathrm{~b}$, c). The Si-doped layers are grown on top of n.i.d. GaN layers with thickness of $250 \mu \mathrm{m}(\mathrm{a}, \mathrm{b})$ and of only $9 \mu \mathrm{m}$ (c). 


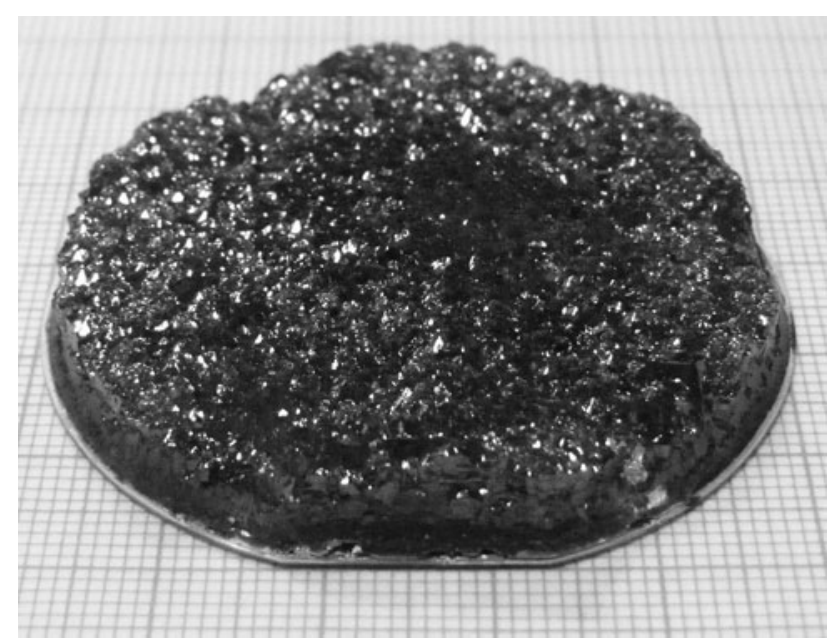

Fig. 8. Si-doped GaN boule of 2 inch diameter and thickness exceeding $6 \mathrm{~mm}$.

This result demonstrates the importance of a sufficiently low TDD before introduction of $\mathrm{Si}$ during the growth of GaN layers in order to avoid tensile strain and cracking. The reduction of the TDD during the growth of $225-\mu \mathrm{m}$-thick n.i.d. GaN (which takes $30 \mathrm{~min}$ in our case) from about $1 \times 10^{9} \mathrm{~cm}^{-2}$ to $2.5 \times 10^{7} \mathrm{~cm}^{-2}$ was found suitable for growth of high-quality Si-doped GaN layers of $1 \mathrm{~mm}$ thickness. Therefore, the growth of the $\mathrm{Si}$-doped GaN layer was prolonged to obtain a $\mathrm{GaN}$ boule with a targeted free electron density of $1 \times 10^{18} \mathrm{~cm}^{-3}$ (Fig. 8).

The GaN boule was sawn into eight $c$-plane slices for comparison with former results for n.i.d. GaN boules. 27 No additional crack formation due to the doping with $\mathrm{Si}$ was found, but the need for further technological improvements already discussed in Ref. 27 is obvious, i.e., prevention of crack propagation from the sapphire substrate, enlargement of the diameter, and optimization of growth conditions to prevent degradation of the growth front in order to increase the crystal length.

\section{CONCLUSIONS}

In this work an approach for Si doping of $\mathrm{GaN}$ in HVPE for the growth of highly conductive, $n$-type $\mathrm{GaN}$ boules which can serve for substrate fabrication is investigated. A commercial vertical reactor, high growth rates above $300 \mu \mathrm{m} / \mathrm{h}$, and diluted dichlorosilane were used for the HVPE growth of $\mathrm{mm}$-thick GaN layers with Si uptakes from $1 \times 10^{18}$ $\mathrm{cm}^{-3}$ to $1.65 \times 10^{19} \mathrm{~cm}^{-3}$. The incorporation of tensile strain was successfully avoided by prior reduction of the dislocation density to $2.5 \times 10^{7} \mathrm{~cm}^{-2}$ by growth of a non-intentionally doped GaN layer of about $225 \mu \mathrm{m}$ on top of a starting GaN/sapphire template. A high electrical activation of about $90 \%$ of the incorporated $\mathrm{Si}$ resulted in free electron concentrations as high as $1.5 \times 10^{19} \mathrm{~cm}^{-3}$. It is shown that the resulting lattice constants are in good agreement with the state-of-the-art lattice constants of undoped $\mathrm{GaN}$ with differences less than $1 \times 10^{-4}$. However, it is also observed that, with increasing $\mathrm{Si}$ incorporation above $2 \times 10^{18} \mathrm{~cm}^{-3}$, the reactivity of screw dislocations is reduced, which results in a slow down of the reduction of threading dislocations with thickness when the density of screw dislocations becomes dominant. Therefore, with the used growth conditions, an upper limit for reasonable use of $\mathrm{Si}$ doping in boule-like GaN HVPE growth of about $4 \times 10^{19} \mathrm{~cm}^{-3}$ can be predicted.

\section{ACKNOWLEDGEMENTS}

The authors gratefully acknowledge Mrs. Helen Lawrenz for her technical assistance in preparation and polishing of the samples. This work was supported by Federal Ministry of Education and Research Projects 01BU0621 and 16BM1202.

\section{REFERENCES}

1. R.M. Farrell, E.C. Young, F. Wu, S.P. DenBaars, and J.S. Speck, Semicond. Sci. Technol. 27, 024001 (2012).

2. K. Fujito, S. Kubo, H. Nagaoka, T. Mochizuki, H. Namita, and S. Nagao, J. Cryst. Growth 311, 3011 (2009).

3. K. Fujito, K. Kiyomi, T. Mochizuki, H. Oota, H. Namita, S. Nagao, and I. Fujimura, Phys. Stat. Sol. (a) 205, 1056 (2008).

4. G.S. Sudhir, Y. Peyrot, J. Krüger, Y. Kim, R. Klockenbrink, C. Kiesielowski, M.D. Rubin, E.R. Weber, W. Kriegseis, and B.K. Meyer, Mater. Res. Soc. Symp. Proc. 482, 525 (1998).

5. A. Cremades, L. Görgens, O. Ambacher, M. Stutzmann, and F. Scholz, Phys. Rev. B 61, 2812 (2000).

6. L.T. Romano, C.G. Van de Walle, J.W. Ager III, W. Götz, and R.S. Kern, J. Appl. Phys. 87, 7745 (2000).

7. C. Kisielowski, J. Krüger, S. Ruvimov, T. Suski, J.W. Ager III, E. Jones, Z. Lilienthal-Weber, M. Rubin, E.R. Weber, M.D. Bremser, and R.F. Davis, Phys. Rev. B 54, 17745 (1996).

8. A.E. Romanov and J.S. Speck, Appl. Phys. Lett. 83, 2569 (2003).

9. A. Dadgar, P. Veit, F. Schulze, J. Bläsing, A. Krtschil, H. Witte, A. Diez, T. Hempel, J. Christen, R. Clos, and A. Krost, Thin Solid Films 515, 4356 (2007).

10. D.M. Follstaedt, S.R. Lee, A.A. Alleman, and J.A. Floro, J. Appl. Phys. 105, 083507 (2009).

11. A. Dadgar, J. Bläsing, A. Diez, and A. Krost, Appl. Phys. Express 4, 011001 (2011).

12. J. Xie, S. Mita, L. Hussey, A. Rice, J. Tweedie, J. LeBeau, R. Collazo, and Z. Sitar, Appl. Phys. Lett. 99, 141916 (2011).

13. A.V. Fomin, A.E. Nikolaev, I.P. Nikitina, A.S. Zubrilov, M.G. Mynbaeva, N.I. Kuznetsov, A.P. Kovarsky, B. Ja, and D.V. Ber Tsvetkov, Phys. Stat. Sol. (a) 188, 433 (2001).

14. A. Usui, M. Shibata, Y. Oshima, Patent US6924159 (2005) 20.

15. E. Richter, Ch. Hennig, U. Zeimer, L. Wang, M. Weyers, and G. Tränkle, Phys. Stat. Sol. (a) 203, 1658 (2006).

16. Y. Oshima, T. Yoshida, K. Watanabe, and T. Mishima, J. Cryst. Growth 312, 3569 (2010).

17. E. Richter, M. Gründer, B. Schineller, F. Brunner, U. Zeimer, C. Netzel, M. Weyers, and G. Tränkle, Phys. Stat. Sol. (c) 8, 1450 (2011).

18. B. Schineller, J. Kaeppeler, and M. Heuken, CS MANTECH Conference, May 14-17, 2007, Austin, Texas, USA, www. csmantech.org/Digests/2007/2007Papers/07a.pdf.

19. M.A. Moram and M.E. Vickers, Rep. Prog. Phys. 72, 036502 (2009).

20. J.H. You, J.-Q. Lu, and H.T. Johnson, J. Appl. Phys. 99, 033706 (2006).

21. J.W. Orton and C.T. Foxon, Semicond. Sci. Technol. 13, 310 (1998). 
22. J.K. Sheu and G.C. Chi, J. Phys.: Condens. Matter 14, R657 (2002).

23. V. Darakchieva, B. Monemar, A. Usui, M. Saenger, and M. Schubert, J. Cryst. Growth 310, 959 (2008).

24. H. Harima, H. Sakashita, and S. Nakashima, Mater. Sci. For. 264-268, 1363-1366 (1998).
25. M. Kuball, Surf. Interface Anal. 31, 987 (2001).

26. S.K. Mathis, A.E. Romanov, L.F. Chen, G.E. Beltz, W. Pompe, and J.S. Speck, Phys. Stat. Sol. A 179, 125 (2000).

27. E. Richter, M. Gründer, C. Netzel, M. Weyers, and G. Tränkle, J. Cryst. Growth 350, 89 (2012). 